\title{
Details and Design of Company CIO Performance Evaluation
}

\author{
ZENG Rong-yu \\ Library \\ Jiangxi University of Science and Technology \\ Ganzhou,P.R.China, 341000 \\ zengry2000@yahoo.com.cn
}

\author{
LIAO Gao-gao \\ Institute of Foreign Language and Foreign Trade \\ Jiangxi University of Science and Technology \\ Ganzhou,P.R.China, 341000 \\ jxlglgg@126.com
}

\begin{abstract}
The article presents the details of company CIO performance evaluation in four dimensions: strategy, execution, revolution and communication. And then, according to the specificity of CIO work, it introduces three main kinds of performance evaluation methods and brings in a concrete performance evaluation design plan_ the Balanced Scorecard Mix with Fuzzy Evaluation Methods.
\end{abstract}

Keywords- company CIO, performance evaluation, evaluation contents, evaluation design

\section{INTRODUCTION}

CIO, the abbreviation of Chief Information Officer who is chiefly in charge of enterprise information department is responsible for the whole information work such as company information management, information system, information technology, etc. CIO, the enterprise senior executive, accomplishes the company strategic objectives by the supporting from information technology. CIO being into strategic circle should be equally constrained by working system and influenced by incentive mechanism with their life and safety being fulfilled. They need higher ego goals and social identity goal. The performance evaluation of CIO mostly is analyzed from the combination of technology and management. Examining CIO's capabilities of deploying and programming information systems, configuring and controlling equipments, training and managing staffs, arranging and supervising projects as well as participating in high-level decision which helps them do a better job and promote the realization of organizational goals[1].

\section{CONTENTS OF PERFORMANCE EVALUATION}

Firstly, the levels to evaluate the work of CIO should be clear, including the enterprises integrating with IT technology and the bring-in opportunities on a strategic side on the basis of CIO job responsibilities, participating more actively in the implementation of great projects such as EPR and CRM on the execution side, reengineering the business process , communicating with upper leaders, departments, subordinates and suppliers. According to these respects, a CIO’s Performance Evaluation can be obtained[2].

\section{A. Evaluation on strategic level}

The CIO being into strategic circle should look on business information management issues with a view of strategic development and be able to make the informationbased strategy, layout and value evaluation as well as develop enterprise information resources. What they are supposed to do consists of four respects: finishing the planning tasks to keep competitive by making use of information technology; playing the role of information system properly to choose and arrange the corresponding information projects; helping companies understand the value of information and process and improve their expectations on information; providing integrated information resources for decision-making and advancing the operation efficiency.

Strategic level evaluation also expands around these aspects. How to evaluate the work and contribution of CIO on a strategic level is determined by the state of correspondence between information development planning and enterprise practical condition; advancement of suggested planning; the orientation effectivity of developing a relationship with customer and realization of value on collaboration of supply chain.

\section{B. Evaluation on operational level}

Nothing can replace specific implementation. There are many specific application projects that call for upper leaders to deal with in the process of enterprise informatization. These projects are called "top managerial project". As we all know, the project presiding by most senior decision-makers has much more chances of success. CIO should go deep into specific projects to take on organizational management responsibilities and play the role of the top leader on enterprise informatization field.

Operational level assessment includes three points. First, the integration effect of information, material and capital flow (determined by the paperless level). Second, the decision information gained for company (help the CIO to make strategic decision). Third, the whole efficiency of IT team , ( includes the construction of internet ,the selection and design of application system and the educational training for staffs).

\section{Evaluation on revolution level}

Evaluation on revolution level is mainly in the advance of business process reengineering and the obtainment of efficiency decision support system and enforcement system. To analyze it with four quantified indicators as following: Firstly, the paperless operation ratio. It can be measured by the using level of electronic document to deliver information and make decision. Secondly, the business flow processing time - a reliable outcome measurement. After the informatization, our system can automatically provide 
workers with the business. Thirdly, the length of business review chain. The decision is reported layer upon layer in the pyramid structure, while informatization has more people sharing informations and more grass-roots staffs can make a decision by themselves. For example, salesman used to get the information of production capacity, raw material and cost through phone and documents, while the informatization system enables salesmen to understand the whole conditions promptly and make sure of the accuracy of decision, which shortens the decision chain from 3 to 5 links to only 2 links. Fourthly, The response time for customer request .Traditional business are greatly dependent on faxes which is responded in days, while information systems can send massages to personnel's mobile phones automatically and the transform can be seen from the changing response time .

\section{Evaluation on communication level}

Business information management, the same as quality control, also needs participation of the entire personnel. Information flow can not work until it integrates with capital and material flow. Communication is the basic requirement to achieve these goals. We can make the evaluation of CIO on communication from three respects. The first one is the informatization training. The details of evaluation can be recorded according to the training times, the range of participation and the training contents for a review. The training result can be obtained through training, testing and the changes of employees' knowledge structure as well as the direct interview. The second one is the understanding of business process. This is the critical part of CIO's communication link. The transform model can be well established to carry out the informatization project by using the process system precisely. CIO, who may be considered as a pioneer of process management, is supposed to help operating department to analyze the business process and set up process management models. Judgements on whether the CIO performed properly depend on the material condition of process management and material delicate degree .The last one is to set up a system based on the value of informatization evaluation. By monitoring the level of information technology, the informatization of enterprise can constantly upgrade, advance with the times.

\section{THE METHOD OF PERFORMANCE EVALUATION}

$\mathrm{CIO}$, as a senior supervisor, whose job duties are mostly to be qualitative and being on macroscopic and administrative level. It is difficult to conduct the evaluation with work performance that can hardly be quantified and most measurements are long-term that is not effective in a short time. In addition, lots of evaluation systems are designed for enterprise pyramid structure, especially for the $80 \%$ of employees who are at the bottom of the pyramid, while few evaluation systems are for decision-makers, especially for the leaders of making enterprise strategic decision. There are three mainly methods below for CIO performance evaluation.

\section{A. Management by objectives(BIO)}

MBO is a way to evaluate the goal achievement though setting expected goals in a certain period. It is actually a progressive cyclic process[3]. As shown in Figure 1.

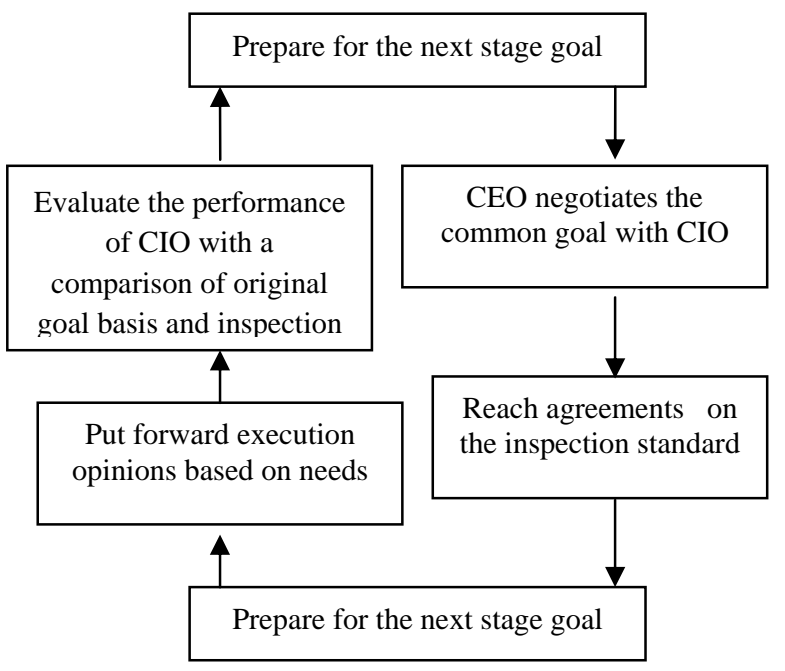

Figure 1. MBO process cycle diagram

When making the evaluation goal, the stress should be laid both on the integrated planning and the using of enterprise information resources with CIO's duties and tasks. To break up the goal of informatization into small ones, the following factors should be taken into consideration: network performance level; computer-networking rate; the coverage rate of informatization of information collecting; office automation system application; informatization level of decision and informatization level of the core business process.

\section{B. 360-degree feedback evaluation}

360-degree feedback evaluation, which differs from the traditional method, is to evaluate performance comprehensively(360-degree) by various estimators. People who are related to the person evaluated are supposed to make an assessment, such as the superiors, the inferiors, the customers, the interior or external clients and the person himself. Then, professional estimators provide a feedback to the person evaluated(as figure 2 shows). This new method definitely improves people's work performance[4]. As shown in Figure 2.

The superiors includes president, director board and CEO. The colleagues includes the chief inspectors from other aspects and the managers and staffs from business department. A comprehensive designed assessment form is used instead of a simple forum. The direction of the evaluation can be in strategy, operational, change and communication level. By making the evaluation records chronically, stably and orderly, the Enterprise CIO can have 
access to opinions around through such an assessment so as to modify his own behavior.

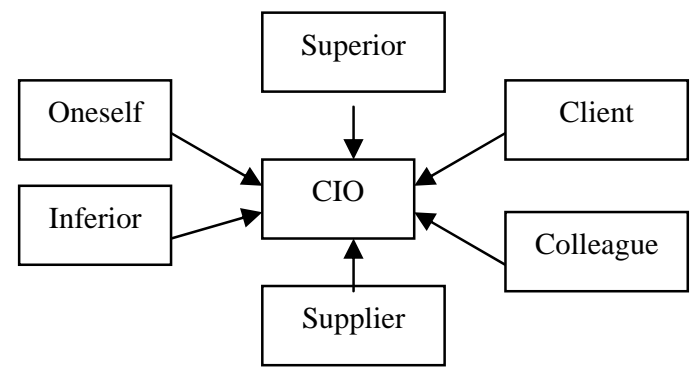

Figure 2. 360-degree Feedback Evaluation Ideograph

\section{The Balanced scorecard}

The balanced scorecard, based on enterprise strategy, integrates all kinds of measures into a whole organically. It includes both financial indexes and operational indicators on customer view, internal process, learning and growth, which enables the organization to track financial results and on the other hand, it also pays a close attention to the intangible assets such as firm's capability improvement and future growth potential. In this way, the enterprise has not only the financial indicators that reflect the "hardware", but also the "software" indexes to win the competition[5]. The CIO's evaulation on strategic, operational, change and communication level is applied as figure 3 below[2]. As shown in Figure 3.

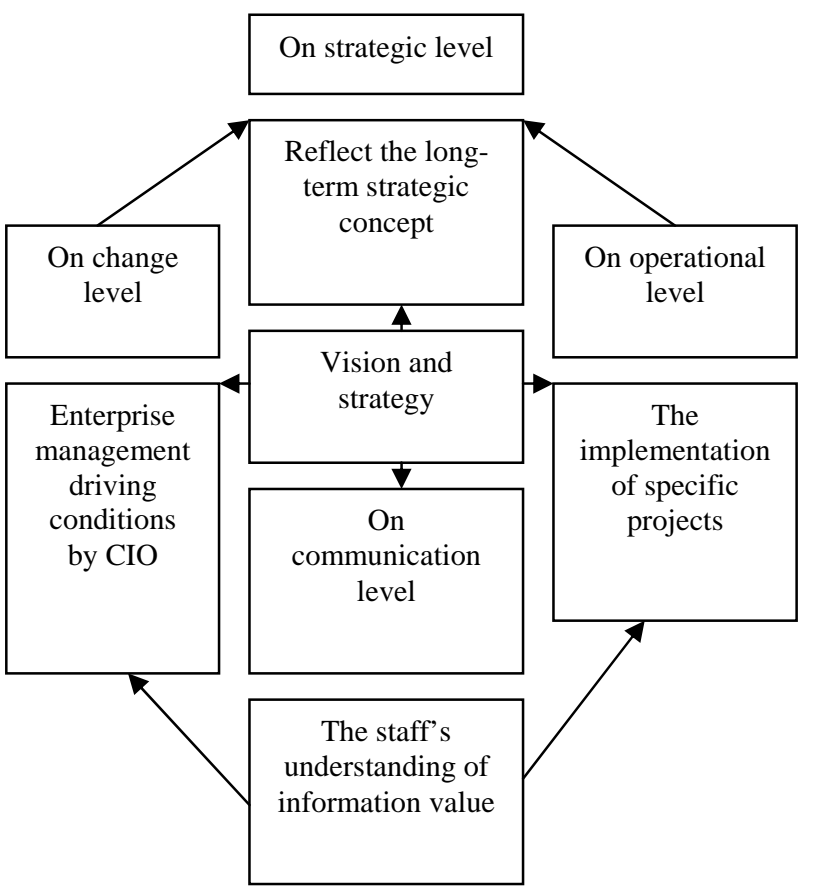

Figure 3. The Balanced Scorecard Ideograph
On strategic level: reflecting the long-term strategic concept is a primary duty of CIO.It can weight to $30 \%$.

On operational level: the implementation of specific projects are being underlined because they are specific battles. It also can weight in $30 \%$.

On change level: $\mathrm{CIO}$ is requested to drive the enterprise management consisted of business process reengineering and the integration of information, capital and material flow.It can weight to $20 \%$.

On communication level: what is demanded is the environment structure and atmosphere of culture. It weight also to $20 \%$.

The balanced scorecard offers a comprehensive evaluation on the work of $\mathrm{CIO}$ and the weight management highlights this position.

\section{PeRformance EVAluation DESign[2][6][7]}

As is known to all ,there are different ways (each has its own character) to evaluate CIO. The balanced scorecard stresses on a balance between shot-term and long-term goal as well as internal and external factor. It gives a comprehensive assessment and highlights the position, which means the balance scorecard is to be a great suggestion in evaluations. However, the diversity of human utilizable value and complexity of human behavior bring difficulties to the valuation measurement of human capital. Therefore, a fuzzy evaluation method in the balanced scorecard evaluation system should be introduced.

\section{A. Steps of forming the balanced scorecard}

(1)Preparation. To define properly for the strategy, execution, revolution and communication involved with CIO .

(2)The first round of access record. Each of senior managers should know about the duties of CIO. The designer of the balanced scorecard asks for comments and suggestions on the aspects of CIO's strategy, execution, revolution and communication with senior managers.

(3)The first round of seminar. Senior managers establish a group to discuss how to form a balanced scorecard which is proper for enterprise CIO. First, discussing the tasks and strategies until the agreement is reached. Second, making sure of the successful factors of CIO. Then, forming a preliminary multifaceted scorecard on CIO performance which at least include 4 to 5 aspects for each .Finally, make a choice on the base of these aspects.

(4)The second round of access record. Asking for opinions of the preliminary multifaceted scorecard contents which formed in first round of the seminar from senior administrators and the director board.

(5)The second round of seminar. This round includes not only senior managers but subordinates and middle managers who are supposed to discuss in group on issues of preliminary multifaceted scorecard to form a relatively complete plan.

(6)The third round of seminar. The aim of this round, with a participation of senior administrative personnel, is to reach a final agreement based on the first two rounds of 
discussion; and is to set specific indicators for evaluation on each aspect and to confirm the completion of preliminary program plan and the achievement of the goal.

(7)Final . Eventually complete the design of balanced scorecard.

(8)Regular inspection and improvement. Put the discussion of information that showed on balanced scorecard between senior managers and division managers and take them into new job duties.

\section{B. Steps of evaluation Implementation:}

(1) Determine the evaluation set $U=\{$ excellent, good, medium, qualified and unqualified

(2) Establish the factor evaluation set $\mathrm{V}=$ \{strategic level, operational level, change level and communication level\}

The weight distribution of evaluation factors: $A=\{0.3,0.3,0.2,0.2\}$

(3) Determine the sub-factor assessment set

$\mathrm{V} 1=$ strategic level $=$ \{complete the task planning; play the role of information systems; help enterprises to know the value of information and process and integrate enterprise information resource, etc. $\}$;

$\mathrm{V} 2=$ operational level $=\{$ integration of information, material and capital flow, information for enterprise decision-making and whole benefits of IT team,etc. $\}$;

$\mathrm{V} 3=$ change level $=\{$ the paperless operation ratio, the business flow processing time, the length of business review chain, the response time for customer request, etc. $\}$;

$\mathrm{V} 4=$ communication level $=$ informatization training, the understanding of business process and to set up a system based on the value of the informatization evaluation,etc.\}.

According to the requirements of different jobs and the importance of sub-factors, score and give the corresponding weights: $0 \leqslant a i j \leqslant 1$, Weight sets $A=$ 。

(4)Construct an evaluation matrix $\mathrm{Ri}=$;

(5) Seek the evaluation matrix with single factor. Calculate the factor membership-rij (as table 1)according to the voting results by assuming that there are 10 people in the assessment group .

As this example, if the sud-factors can be further subdivided, subdivide the whole benefits of IT team on operational level into: computer network construction, application system selection and design, staff education training, etc. The value of rij obtains from the next level factor evaluation matrix calculation.
TABLE I. SINGLE FACTOR EVALUATION MEMBERShIP

\begin{tabular}{|l|c|c|c|c|c|c|}
\hline $\begin{array}{c}\text { evaluati } \\
\text { on }\end{array}$ & $\begin{array}{c}\text { excell } \\
\text { ent }\end{array}$ & good & $\begin{array}{c}\text { medi } \\
\text { um }\end{array}$ & $\begin{array}{c}\text { qualifi } \\
\text { ed }\end{array}$ & $\begin{array}{c}\text { unqual } \\
\text { ified }\end{array}$ & total \\
\hline $\begin{array}{l}\text { the } \\
\text { number } \\
\text { of voters }\end{array}$ & 2 & 4 & 3 & 1 & 0 & 10 \\
\hline rij & 0.2 & 0.4 & 0.3 & 0.1 & 0 & 1 \\
\hline
\end{tabular}

(6) Seek the comprehensive evaluation matrix $\mathrm{Bi}=\mathrm{Ai} \cdot \mathrm{Ri}$

(7)To find the value of $\mathrm{B}$ from the total evaluation matrix $\mathrm{B}=$.

(8)Seek for the System evaluation matrix $\mathrm{C}=\mathrm{A} \cdot \mathrm{B}=$

(9)Find out the biggest bi value - the maximum degree of membership as the evaluation standard according to the operational results .

Of course, the proper expression of performance evaluation results can be selected to raise the level. There are two kinds of expression.

Qualitative expression: express the results of CIO evaluation in four dimensions through comments qualitatively.

(2)Quantitative expression: using the grade or score value to express the results.

a)The expression in grade. There are three grades fourth, fifth and seventh grade in general. For example, in the fifth grade: A,B,C,D,E each represents for "excellent, good, medium, qualified and unqualified" and each grade can be subdivided into several level. Take "good" for instance, it can be subdivided into "on good, in good and under good”.

b)The expression in fraction. It usually use the hundredmark system.

The evaluation system is established in a comprehensive way, which also highlights the CIO position and reflects the CIO work effect roundly. The application of fuzzy mathematics calculation model aims to determine the membership of performance indicators decreases influence on subjective factors in the assessment. Therefore, the evaluation results become more objective and fair. It is compatible to be applied to the performance evaluation of CIO who acts as both a senior manager and information expert.

\section{REFERENCES}

[1] XuHao,Wen-Juan Yang.Research on Enterprise CIO Performance Evaluation[J].Science Technology and Industry,2008(7).

[2] WangMuQing, XiaJingHua, WenBo.Leading Enterprise Informatization - CIO workbook[M].Beijing:Electronic Industry Press,2004.

[3] WangHao. Human Resource Management[M],Beijing: Chinese Press,2003.

[4] RaoZheng, Flavien .Take KPI As The Core Of Performance Management[M],Beijing: Renmin University of China press,2003.

[5] Jian-Feng Peng. Introduction to Human Resource Management[M],Shanghai: Fudan university press, 2003.

[6] MaQian jie,Ben-Hai Yu.Theory and Method of Information Resources Evaluation [M],Beijing:Economic Science Press ,2002. 
